\title{
Flick Gesture Interaction in Augmented Reality: AR Carrom
}

\author{
Apurv Varshney \\ University of California, Santa Barbara \\ apurv@ucsb.edu \\ Richa Wadaskar \\ University of California, Santa Barbara \\ richa_wadaskar@ucsb.edu
}

\author{
Justin Nilsen \\ University of California, Santa Barbara \\ jnilsen@ucsb.edu \\ Misha Sra \\ University of California, Santa Barbara \\ sra@ucsb.edu
}

\begin{abstract}
Gestural input can make augmented reality applications feel natural and intuitive. While gestures used as simple input controls are common, gestures that interact directly with the virtual objects are less so. These interaction gestures pose additional challenges since they require the application to make many interpretations about the hand in the camera's field of view such as depth, occlusion, size, and motion. In this work, we describe and propose a flick gesture control mechanic that estimates force and direction from a baseline pinch gesture. We demonstrate the gesture through an example implementation of an AR version of a game called Carrom in which we use our flick mechanic to dynamically interact with the virtual Carrom striker.
\end{abstract}

\section{CCS CONCEPTS}

- Human-centered computing $\rightarrow$ Human computer interaction (HCI); Interaction paradigms; Mixed / augmented reality;

\section{KEYWORDS}

carrom, augmented reality, boardgame, hand gesture recognition, flick gesture, depth occlusion

\section{ACM Reference Format:}

Apurv Varshney, Justin Nilsen, Richa Wadaskar, and Misha Sra. 2021. Flick Gesture Interaction in Augmented Reality: AR Carrom. In The Adjunct Publication of the 34th Annual ACM Symposium on User Interface Software and Technology (UIST '21 Adjunct), October 10-14, 2021, Virtual Event, USA. ACM, New York, NY, USA, 3 pages. https://doi.org/10.1145/3474349.3480229

\section{INTRODUCTION}

Many games that are primarily tactile - like billiards, ping pong, or Carrom - require players to manipulate the game objects with their hands. Until recently, online versions of these games were limited to traditional modes of input like a mouse and keyboard, which are insufficient to recreate the variety of hand and object-based input mechanisms used in these games. Recent advancements in augmented reality (AR) and especially in gesture recognition have

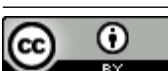

This work is licensed under a Creative Commons Attribution International 4.0 License.

UIST '21 Adjunct, October 10-14, 2021, Virtual Event, USA

(c) 2021 Copyright held by the owner/author(s).

ACM ISBN 978-1-4503-8655-5/21/10.

https://doi.org/10.1145/3474349.3480229 begun to bridge that gap, enabling gesture interaction with AR objects themselves to better simulate the tactile play experiences.

A challenging problem for hand gesture recognition is interactivity with virtual AR elements. Simple gestures as input (ex: a thumbs-up gesture to the camera triggers a pop-up menu) are common, unlike gestures that interact directly with virtual content (ex: sending a virtual object flying by flicking it), which pose several technical challenges. In this work, we describe a new flick gesture mechanic that estimates the flick's force and direction, to interact dynamically with virtual objects. We introduce our mechanic by demonstrating it in an AR Carrom game, a popular tabletop Indian game wherein players flick a striker on a board attempting to sink all of their pieces into corner pockets. Our interaction mechanic includes (1) a gesture recognition system based on the Manomotion Community Edition SDK [1] to determine when the user makes a flick gesture with their index finger and thumb using either hand; (2) direction and estimation using the changing location of a point of interest (POI) of the flick, and (3) occlusion of the hand over the Carrom board using the ARCore Depth API.

Prior work has extensively explored freehand gestures for AR and VR [2-4] and consumer AR apps have attempted to recreate physical/tactile AR games. For example, AR Jenga [5] uses the device touchscreen or AR chess [6] uses the pinch gesture to move the pieces. However, these AR games are either unable to recreate the natural hand gestures used to play the physical versions of their respective games or require external sensors and physical boards, negating the advantages mobile AR games can offer in terms of flexibility in when and where they can be played. Currently, there are two other AR Carrom applications available for consumers. AR Pocket Carrom [7] is a mobile app that allows the user to navigate the Carrom board in real 3D space using their phone. However, the app has no gesture detection and consists entirely of screen-based interaction. Augmented-Reality-Carrom-Board [8] offers gesture detection - a step up from AR Pocket Carrom - but requires the use of external hardware (a thick wool glove for mocap connected to wires). Our contribution, AR Carrom, provides a novel flick gesture with correct occlusion using camera-based input and without the need for extra hardware.

\section{SYSTEM OVERVIEW}

Carrom's rules are similar to that of Billiards. Players compete by striking the striker to pocket their pieces and score points. We implemented this striking motion using a flick gesture interaction that allows players to flick the "striker" to hit other pieces much like a cue ball does in billiards. Figure 1 shows app screenshots. 


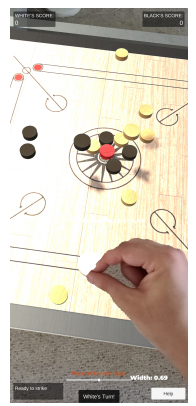

(a)

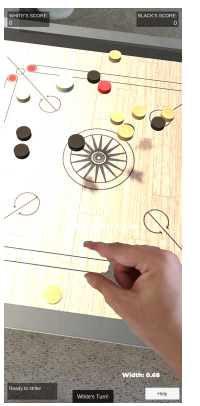

(b)

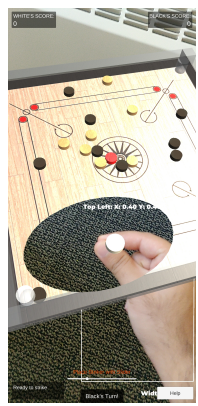

(c)

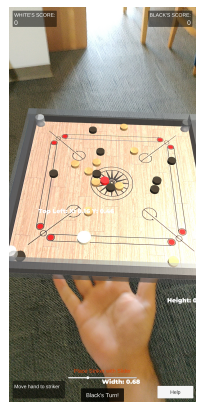

(d)
Figure 1: (a)\&(b): Carrom game in play with the user making a flick gesture (c): Early Occlusion attempt (d): ARCore Depth API on Android w/o ToF sensor.

\section{IMPLEMENTATION}

The subsections below detail the core components of our system.

\subsection{Gesture Recognition}

For hand tracking and gesture recognition, we utilized the Manomotion Community Edition SDK [1]. This SDK provides several useful hand attributes such as palm center position, point of interest (POI), and a rectangular bounding box around the detected hand. Manomotion Community SDK's main limitation is its restriction to a set of preset gestures, which does not include the flick gesture.
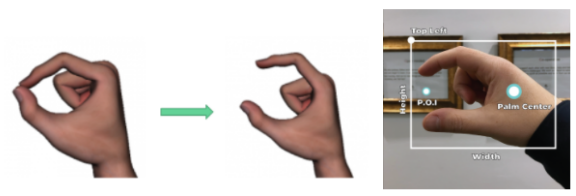

Figure 2: Flick gesture recognition \& bounding box

The key gesture necessary to play our Carrom game is the flick gesture seen in Figure 2. The user must flick the striker using either hand in a particular direction with the force they find appropriate. For the gesture to flick the striker, we constrained the striker to be inside the bounding box around the hand. We modified Manomotion's drop gesture (the pinch gesture - so named because of its intended application to drag-and-drop items in the virtual world by pinching and releasing) to store hand position information (POI location in $3 \mathrm{D}$ space) before and after the flick (drop gesture). Manomotion provides the POI for the closed and open pinch. In a closed pinch, the POI is the intersection of the thumb and pointer finger. An open pinch POI can also be seen in Figure 2. Figure 2 also shows an example of a bounding box enclosing a player's hand, along with some hand properties provided by Manomotion.

\subsection{Force Direction and Estimation}

To calculate the direction that the striker should be sent in, we stored the POI of the closed pinch and calculated the direction from this point to the POI of the open pinch gesture. Since players are expected to flick with their hands parallel to the board (along the XZ plane), we zero-out the $y$-coordinate of the force direction vector to make it parallel with the board, giving us the intended direction of the user's flick. While this technique was not $100 \%$ accurate, it is a good approximation, at least insofar as Manomotion can correctly guess the depth of the gesture's POI and, using the camera's current orientation in world space, translate those $2 \mathrm{D}$ screen coordinates to $3 \mathrm{D}$ virtual world space coordinates through raycasts. Those tasks were performed by Manomotion, meaning the accuracy of our direction technique is largely guided by the accuracy of the underlying plugin used to obtain gesture information.

To calculate the flick force, we first considered using the magnitude of the vector from the closed pinch POI to the open pinch POI. However, this would be inaccurate because the closer the hand is to the camera, the larger the magnitude of the vector. Instead, we decided to utilize the percentage change in the bounding box surrounding the user's hand, multiplied with a maximum force value (which we chose heuristically - future research may discover an optimal value) which gave the striker a natural forward force.

\subsection{Occlusion}

Accurate depth measurement of objects in a camera's image, and properly occluding such objects in the virtual space, is a challenging and computationally intensive task for real-time AR applications. In Carrom, the player's hand needs to occlude (be over) the Carrom board, but the background (e.g., table, floor) behind the hand must in turn be occluded by the Carrom board. At present, only the newest devices with ToF (Time of flight) or LIDAR (Light Detection and Ranging) sensors support such functionality through ARCore's Depth API. Hence, we implemented our system on a Samsung S20+ phone (which contains a ToF depth sensor) to achieve intended occlusion as seen in Figure 1a \& 1b. Prior to that, we attempted to simulate occlusion with a sphere (Figure 1c) which followed the hand's POI and used a specialized transparent shader. The sphere was rendered before the Carrom board but after the Carrom pieces, solving the occlusion problem on a functional level but aesthetically detracting from the play experience. Using no occlusion at all (Figure 1d) had the opposite problem - the hand was hidden behind the virtual Carrom board.

\section{CHALLENGES AND LIMITATIONS}

We faced several challenges during development, mainly in calculating the force and direction of the flick, and hand occlusion. The flick direction's accuracy is limited by the maturity of the depth API we used, the flick gesture only detects the thumb and pointer finger, and our force estimation doesn't account for the time interval over which the flick occurs. We hope to extend these by building our own gesture detection system in the future. AR Foundation supports human segmentation only in newer iOS devices, and ARCore's Depth API only works on newer Android devices with a depth sensor. We hope human occlusion for Android is released more broadly as using the currently available environmental occlusion caused a significant delay in rendering each frame. 


\section{CONCLUSION}

In this work, we presented a flick gesture mechanic with proper occlusion and demonstrated it through the game AR Carrom. Our contribution uses freehand gesture recognition to estimate force, direction, and hand position in the virtual world to interact with the Carrom striker, all without the need for any external hardware.

\section{ACKNOWLEDGMENTS}

We thank Mengyu Chen for his help with app deployment and testing.

\section{REFERENCES}

[1] Manomotion Community Edition SDK, https://www. manomotion. com/documentation/
[2] Yang, L. I., Huang, J., Feng, T. I. A. N., Hong-An, W. A. N. G., \& Guo-Zhong, D. A. I. (2019). Gesture interaction in virtual reality. Virtual Reality \& Intelligent Hardware, 1(1), 84-112.

[3] Billinghurst, Mark, Tham Piumsomboon, and Huidong Bai. "Hands in space: Gesture interaction with augmented-reality interfaces." IEEE computer graphics and applications 34.1 (2014): 77-80.

[4] Buchmann, V., Violich, S., Billinghurst, M., \& Cockburn, A. (2004, June). FingARtips: gesture based direct manipulation in Augmented Reality. In Proceedings of the 2nd international conference on Computer graphics and interactive techniques in Australasia and South East Asia (pp. 212-221).

[5] Range, Free. "Jenga ${ }^{\circledR}$ AR." App Store, 27 Jan. 2021, apps.apple.com/us/app/jengaar/id1547477000.

[6] Bikos, Marios \& Itoh, Yuta \& Klinker, Gudrun \& Moustakas, Konstantinos. (2015). An Interactive Augmented Reality Chess Game Using Bare-Hand Pinch Gestures. 10.1109/CW.2015.15.

[7] Muhammad Salman. "AR Pocket Carrom Player." AppAdvice, 23 Apr. 2019, https://appadvice.com/app/ar-pocket-carrom-player/1340074234.

[8] Augmented-Reality-Carrom-Board,

ht tps://gi thub.com/m-shahbaz-kharal/Augmented-Reality-Carrom-Board 